\title{
Erratum: Minimax strategy in quantum signal detection with inconclusive results [Phys. Rev. A 88, 032314 (2013)]
}

\author{
Kenji Nakahira $\odot$, Kentaro Kato, and Tsuyoshi Sasaki Usuda
}

Q (Received 26 December 2020; published 11 January 2021)

DOI: 10.1103/PhysRevA.103.019903

The sentence including Eq. (6), which starts with "From the minimax theorem ...," should read as follows: "Assume that $\Pi^{\star} \in \mathcal{M}$ and $\xi^{\star} \in \mathcal{X}$ satisfy

$$
\max _{\Pi \in \mathcal{M}} P_{\mathrm{C}}\left(\xi^{\star}, \Pi\right)=P_{\mathrm{C}}\left(\xi^{\star}, \Pi^{\star}\right)=\min _{\xi \in \mathcal{X}} P_{\mathrm{C}}\left(\xi, \Pi^{\star}\right) .
$$

From the minimax theorem (e.g., [23]), such $\left(\xi^{\star}, \Pi^{\star}\right)$ exists and we have $[4,7]$

$$
\min _{\xi \in \mathcal{X}} \max _{\Pi \in \mathcal{M}} P_{\mathrm{C}}(\xi, \Pi)=P_{\mathrm{C}}\left(\xi^{\star}, \Pi^{\star}\right)=\max _{\Pi \in \mathcal{M}} \min _{\xi \in \mathcal{X}} P_{\mathrm{C}}(\xi, \Pi) . ”
$$

The sentence including Eq. (11), which starts with "From the minimax theorem ...," should read as follows: "Assume that $\Pi^{\star} \in \mathcal{M}_{p}$ and $\xi^{\star} \in \mathcal{X}$ satisfy

$$
\max _{\Pi \in \mathcal{M}_{p}} P_{\mathrm{CI}}\left(\xi^{\star}, \Pi\right)=P_{\mathrm{CI}}\left(\xi^{\star}, \Pi^{\star}\right)=\min _{\xi \in \mathcal{X}} P_{\mathrm{CI}}\left(\xi, \Pi^{\star}\right) .
$$

From the minimax theorem, such $\left(\xi^{\star}, \Pi^{\star}\right)$ exists and we have

$$
\min _{\xi \in \mathcal{X}} \max _{\Pi \in \mathcal{M}_{p}} P_{\mathrm{CI}}(\xi, \Pi)=P_{\mathrm{CI}}\left(\xi^{\star}, \Pi^{\star}\right)=\max _{\Pi \in \mathcal{M}_{p}} \min _{\xi \in \mathcal{X}} P_{\mathrm{CI}}(\xi, \Pi) . "
$$

The sentence including Eq. (12), which starts with "The minimax theorem also ensures ...," should be removed. 